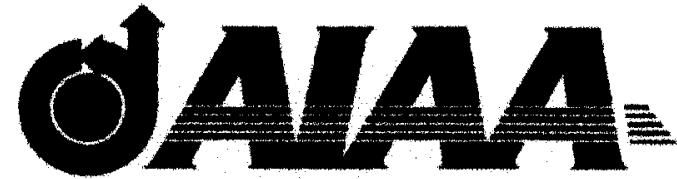

\title{
A01-34241
}

\section{AIAA 2001-3507 \\ Considerations on the Role of the Hall Current in a Laboratory-Model Thruster}

J.M. Haas and A.D. Gallimore

Plasmadynamics and Electric Propulsion Laboratory

Department of Aerospace Engineering

The University of Michigan

Ann Arbor, MI

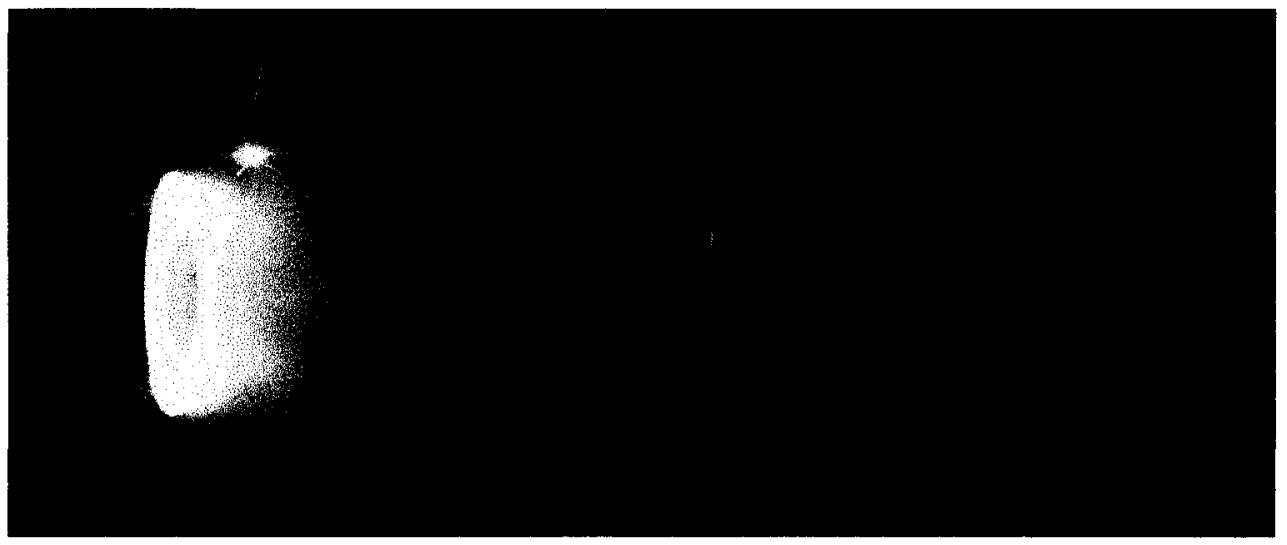

$37^{\text {th }}$ Joint Propulsion Conference \& Exhibit

$$
\begin{gathered}
8-11 \text { July } 2001 \\
\text { Salt Lake City, Utah }
\end{gathered}
$$

For permission to copy or to republish, contact the American Institute of Aeronautics and Astronautics, 1801 Alexander Bell Drive, Suite 500, Reston, VA, 20191-4344. 
AIAA 2001-3507

\title{
Considerations on the Role of the Hall Current in a Laboratory-Model Thruster
}

\author{
James M. Haas" and Alec D. Gallimore ${ }^{\dagger}$ \\ Plasmadynamics and Electric Propulsion Laboratory \\ Department of Aerospace Engineering \\ The University of Michigan \\ College of Engineering \\ Ann Arbor, MI 48109 USA
}

\begin{abstract}
Hall current magnitude and spatial distribution are presented for the plasma discharge in the University of Michigan/Air Force Research Laboratory P5 $5 \mathrm{~kW}$ laboratory-model Hall thruster. The data are calculated from direct, probe-based measurements of the electric field, static magnetic field, and charged particle number density. Thruster discharge voltage was fixed at $300 \mathrm{~V}$ and two current levels investigated: $5.4 \mathrm{~A}(1.6 \mathrm{~kW})$ and $10 \mathrm{~A}(3 \mathrm{~kW})$. The results indicate that, for both cases, the bulk of the Hall current is confined to a region centered several millimeters upstream of the exit plane and is asymmetric about the centerline of the discharge channel. At $1.6 \mathrm{~kW}$, the axial plasma potential drop occurs over a much shorter distance, resulting in a more sharply peaked Hall current zone, as compared to the $3 \mathrm{~kW}$ case. Comparison of the Hall current and ion number density distribution suggests that the azimuthal electron drift may contribute significantly to the ionization process in the discharge channel. Integration of the Hall current over the entire discharge volume yields total current values that are a factor of 3.5 - 4.6 times larger than the discharge current. Estimates of the self-magnetic field induced by the drifting electrons indicate no significant modification to the applied magnetic field during thruster operation, at the power levels considered. Using the Hall current density distribution derived from probe measurements, the electromagnetic body force on the ions was calculated and compared to measured engine thrust for both power levels.
\end{abstract}

\section{INTRODUCTION}

The Hall thruster, also referred to as a Closed Drift Thruster (CDT), is a coaxial device in which orthogonal electric and magnetic fields are employed to ionize an inert gas, such as xenon, and accelerate the resulting ions to produce thrust. A direct consequence of the crossed field configuration is a bulk azimuthal motion of the electrons in the Hall thruster discharge. This motion is the cumulative effect, averaged over many gyroradii, of small variations induced in each cycle of the electrons cyclotron motion by the electric field. The electrons may effect many rotations around the circumference of the discharge channel before undergoing a collision with the channel wall or another particle, owing to its annular configuration; hence the oftused designation "closed drift".

The azimuthal drift velocity can be calculated from the known electric and magnetic field magnitude and is defined by Equation 1.

$$
V_{E \times B}=\frac{(\mathbf{E} \times \mathbf{B})_{\theta}}{B^{2}}=\frac{E_{z} B_{r}-E_{r} B_{z}}{B^{2}} \quad \text { Eq. } 1
$$

The axial, radial, and azimuthal directions are denoted by the subscripts $z, r$, and $\theta$, respectively. It is generally assumed that the axial magnetic field, $B_{z}$, and radial electric field, $E_{r}$ are small and thus the drift velocity reduces to the simplified form shown in Equation 2.

$$
V_{E \times B}=\frac{E_{z}}{B_{r}}=-\frac{1}{B_{r}} \frac{d \phi}{d z}
$$

The electron drift velocity can then be combined with the electron number density to determine the Hall current density according to Equation 3 ,

*Currently Research Scientist, Air Force Research Laboratory, Edwards AFB.
†Associate Professor, Associate Fellow AIAA.

Copyright $(\mathcal{O} 2001$ by James M. Haas

Published by the American Institute of Aeronautics and Astronautics with Permission. 


$$
j_{E x B}=n_{e} q V_{E x B}
$$

where $q$ is the elementary electron charge. Throughout this analysis $n_{i}$ and $n_{e}$ will be used interchangeably due to the assumption of quasineutrality.

The objective of this research is to quantify the magnitude and spatial structure of the Hall current in a laboratorymodel Hall thruster. This is accomplished through the application of Equations 2 and 3 to direct measurements of plasma potential, using a floating emissive probe, and electron number density, using a double Langmuir probe. The results are then used in conjunction with the density and temperature measurements to examine the roll of the Hall current in the thruster discharge.

\section{FACILITIES AND DIAGNOSTICS}

\section{Thruster}

The thruster used is the University of Michigan/Air Force Research Laboratory P5 $5 \mathrm{~kW}$ laboratory-model Hall thruster. The P5 incorporates a Lanthanum Hexaboride $\left(\mathrm{LaB}_{6}\right)$ cathode. This thruster was developed specifically to provide extensive diagnostic access to the discharge chamber. Thrust, specific impulse, and efficiency have been characterized thoroughly and compare favorably with commercially available state-of-the-art thrusters. Performance characteristics and plasma parameter profiles in the plume have been reported in a previous work. ${ }^{1}$

Thruster discharge voltage was fixed at $300 \mathrm{~V}$ for all experiments. Two discharge current levels were considered: $5.4 \mathrm{~A}(1.6 \mathrm{~kW})$ and $10 \mathrm{~A}(3 \mathrm{~kW})$. These corresponded to anode mass flow rates of $63 \mathrm{sccm}$ and 112 sccm, respectively. Cathode mass flow rate remained constant at $6 \mathrm{sccm}$.

\section{Vacuum Chamber}

All experiments were conducted in the University of Michigan's $6 \mathrm{~m}$ diameter by $9 \mathrm{~m}$ long Large Vacuum Test Facility (LVTF). The pumping system consists of seven CVI model TM-1200 Re-Entrant Cryopumps providing a measured xenon pumping speed of $240,000 \mathrm{l} / \mathrm{s}$. The ultimate base pressure of the facility less than $2 \times 10^{-7}$ Torr. Four of the seven cryopumps were used for these tests. The resulting operating pressures for this experiment were $5.5 \times 10^{-6}$ Torr and $9.6 \times 10^{-6}$ Torr when corrected for Xenon and corresponded to discharge currents of 5.4 $\mathrm{A}$ and 10.0
A, respectively. Details of the facility are presented elsewhere. ${ }^{1}$

\section{HARP}

The emissive and double Langmuir probes are positioned inside the Hall thruster discharge chamber using the PEPL HARP (High-speed Axial Reciprocating Probe) system. The HARP system allows the probe to be inserted into, and removed from, the thruster in less than $100 \mathrm{~ms}$. This short residence time allows measurements to be made with very little perturbation to thruster operation. The extent of thruster perturbation is determined by monitoring the discharge current during probe movement. Use of the emissive and Langmuir probes caused a slight perturbation in the discharge current but this remained less than $10 \%$ of the nominal discharge current value during all measurements. Probe data were collected during both insertion and removal of the probe and averaged to yield the values used in calculating the Hall current.

The HARP system provided only the axial positioning of the probes. In order to generate the two-dimensional (2D) mapping inside the discharge channel, the thruster was mounted to a custom built, two-axis positioning stage developed by New England Affiliated Technologies (NEAT). This allowed the thruster to be moved radially between each axial probe sweep. The 2D data collection domain is shown in Figure 1.

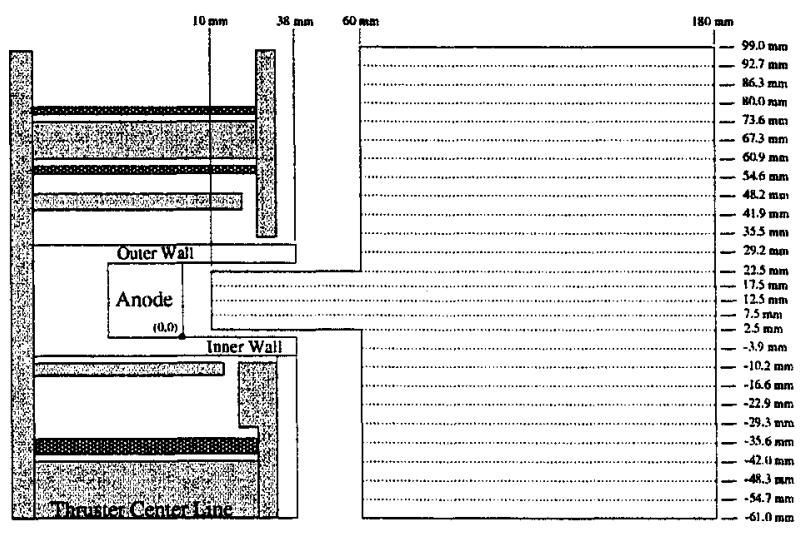

Figure 1. Data collection domain. The internal area mapped begins $10 \mathrm{~mm}$ downstream of the anode face and extends to $60 \mathrm{~mm}$. Radial, data are collected starting $\mathbf{2 . 5} \mathbf{~ m m}$ from the inner wall and ending $\mathbf{2 . 5}$ $\mathrm{mm}$ from the outer wall. The large external domain extends radially from the thruster centerline to the outer pole piece and $180 \mathrm{~mm}$ downstream.

The internal area mapped begins approximately $10 \mathrm{~mm}$ from the anode face, extends $2 \mathrm{~cm}$ downstream of the exit plane, and comes to within $2.5 \mathrm{~mm}$ of the inner and outer walls of the discharge chamber. Data obtained in the internal and near-field areas will be the main focus of 
subsequent analyses. The larger area extends axially from 2 to $14 \mathrm{~cm}$ downstream of the exit plane and radially from the thruster centerline to the outer edge of the outer front pole piece. The gap in front of the anode and the face of the thruster is due to limitations of the HARP. The HARP experienced occasional overshooting of its target position and as a result a large safety margin was required to avoid hitting the thruster and destroying the probe.

\section{Hall Probe}

The magnetic field structure inside the P5 was measured using a Hall sensor. Benchtop magnetic field measurements were made using a commercial system manufactured by F. W. Bell. The system consisted of a Hall sensor connected to a Gaussmeter, which provided a fixed current signal and measured the resulting voltage drop across the output terminals of the sensor in the presence of a magnetic field. The measured voltage was then converted to its corresponding magnetic field magnitude using the manufacturer supplied, NIST traceable, calibration factor. The sensor itself was mounted to a two-axis positioning system to allow twodimensional mapping of the radial and axial magnetic field magnitude in the discharge channel of the P5.

Magnetic field uncertainty was specified by the manufacturer to be less than $1 \%$. Based on the size of the Hall sensor, there was an uncertainty in the probe axial position of $\pm 1 \mathrm{~mm}$ and the radial position of less than 1 $\mathrm{mm}$, for the radial magnetic field measurements. Vacuum axial magnetic field values were obtained with a Hall sensor similar in size to that used for the radial measurements but rotated 90 degrees such that its face was normal to the thruster axis. For the axial field measurements, the axial uncertainty was less than $1 \mathrm{~mm}$ while the radial uncertainty was approximately $\pm 1 \mathrm{~mm}$.

\section{Emissive Probe}

Inside the Hall thruster, the flux of high-energy particles sputters and/or heats electrostatic probe material causing it to ablate. Local plasma characteristics are then affected through emission of relatively cool probe material. These local variations propagate through the plasma, perturbing thruster operation, making it imperative that the residence time of any diagnostic inside the Hall thruster be minimized. It is for this reason that the floating emissive probe was chosen to make plasma potential measurements inside the P5. The floating emissive probe provides a direct measure of plasma potential without the requirement of a voltage sweep or data reduction operations, as is the case for both the standard emissive probe and the Langmuir (electron-collection) probe.
Figure 2 shows a schematic of the emissive probe construction. The emitting portion of the probe was a filament made from $0.127 \mathrm{~mm}$ diameter tungsten wire. The ends of this filament were inserted approximately 76 $\mathrm{mm}$ down a $152 \mathrm{~mm}$ length of double bore alumina tubing along with 30 AWG copper wire leads. The alumina insulator had an outer diameter of $1.5 \mathrm{~mm}$.

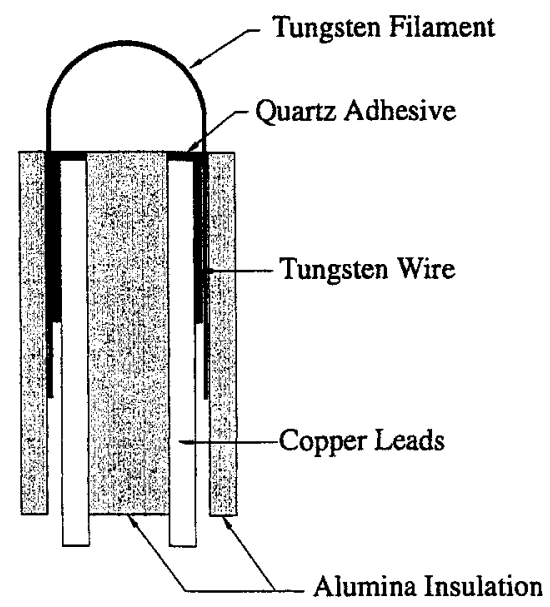

Figure 2. Schematic of the emissive probe. The probe was constructed using $0.127 \mathrm{~mm}$ diameter tungsten wire for the emitting filament, insulated by $1.5 \mathrm{~mm}$ diameter alumina tubing.

Once the tungsten filament and copper leads were in place additional, shorter lengths of tungsten wire were inserted into the alumina tubing to provide a tight fit and guarantee good contact between the tungsten and copper wires. The width and height of the filament when mounted in the alumina insulator was approximately $1 \mathrm{~mm}$. The probe was oriented such that the alumina insulator was aligned with the axis of the thruster and the plane of the loop of the probe filament was perpendicular to the thruster radial direction.

While the floating emissive probe offers many advantages, there are limitations that must be taken into account. Specifically, the presence of a magnetic field and large density gradients may result in space-charge effects, causing significant variation between the potential of the emitting probe and the actual plasma potential. In the case of the P5, the magnetic field is sufficiently weak that space-charge effects can be avoided by sizing the probe such that the probe wire diameter is much less than the electron gyroradius. Hershkowitz ${ }^{1}$ indicated this condition as shown in Equation 4

$$
B<\frac{4.8\left(T_{e V}\right)^{1 / 2}}{d} \quad \text { Eq. } 4
$$


where $T_{e V}$ is the electron temperature in $\mathrm{eV}, d$ is the emitting filament diameter in $\mathrm{cm}$ and $B$ is the magnetic field in Gauss. The emissive probe used in the P5 had a diameter of $0.0127 \mathrm{~cm}$. The electron temperature varied between 10 and $40 \mathrm{eV}$ in the channel (based on double Langmuir probe measurements made in the P5), with higher values generally corresponding to areas of large magnetic fields. Using the minimum electron temperature, the worst case condition is $\mathrm{B} \ll 1200 \mathrm{G}$. The magnetic field in the P5 peaked at $200 \mathrm{G}$, indicating that spacecharge effects were negligible.

Figure 3 shows a schematic of the probe circuit.

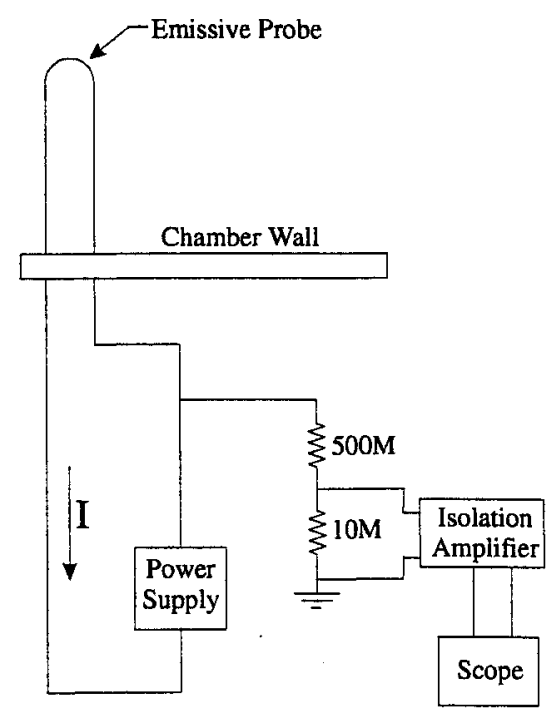

Figure 3. Floating emissive probe circuit. Current is applied to the electrode (generally small gauge tungsten wire), heating it to the point where electrons are thermionically emitted. When heated sufficiently, the emitted electrons essentially neutralize the sheath. At this point, the probe will float at the local plasma potential.

\section{Double Langmuir Probe}

Langmuir probes provide one of the best means for making spatially resolved measurements of electron temperature and number density in plasmas. While the basic probe theory in an ideal plasma is quite simple to implement, the plasma characteristics in the discharge channel of the Hall thruster create significant difficulties in collecting and interpreting probe data. Therefore, great care must be taken in choosing the shape, size, and orientation of the probe due to the presence of streaming ions, high plasma potentials and potential gradients, and large radial magnetic fields. Fortunately, many of these effects can be made negligible with proper consideration.

Langmuir probes are generally classified according to two parameters: the number and the shape of the electrodes. Planar, cylindrical, and spherical probes with one to four electrodes have been used in a wide range of plasmas and the theory of their operation is extensive. Spherical probes were immediately rejected for this experiment because of the difficulty in their construction, particularly of sizes small enough to provide good spatial resolution inside the thruster. Both planar and cylindrical probes are easily constructed at very small sizes and can be configured as single, double or triple probes.

Recalling that the probe is inserted and removed from the thruster in approximately $100 \mathrm{~ms}$, the triple probe is very attractive because it does not require a voltage sweep ${ }^{2}$. It needs only a fixed voltage between two electrodes while the third floats. However, in this case size again becomes an issue. Because of the wide range of parameters in the thruster, the Debye length can grow considerably, necessitating a large separation between electrodes. This leads to a large overall probe size that can severely perturb the thruster and reduces the resolution of the measurement.

Of the single and double probe configurations, the double probe provides several advantages in the Hall thruster. The single probe potential is biased with respect to some fixed reference; generally electrical or chamber ground. The bias range starts highly negative (ion saturation) and extends to the plasma potential (electron saturation). In the Hall thruster, plasma potential ranges from tens of volts in the near-field region to several hundreds of volts (discharge voltage) near the anode ${ }^{3}$. This greatly complicates the experimental setup as the sweep voltage range is quite large and changes dramatically throughout the discharge chamber. This need to bias the probe at or beyond the plasma potential also creates serious perturbation problems. The electron saturation current can be several orders of magnitude larger than the ion saturation current and drawing this much current from the plasma can cause serious perturbations to thruster operation.

The double probe eliminates most of the problems associated with the single probe. A voltage sweep is still required but it is between two electrodes which float as a whole, one above the floating potential and one below. The electron current to a given electrode is limited by the ion saturation current of the other electrode and, because the probe is floating, the net current to the probe is zero. This results in very little perturbation to the plasma. Also, because the current is limited to the ion saturation current, a much smaller voltage sweep range is required.

The decision to use a double probe dictated the final choice of a cylindrical geometry. As stated above, the current collected by a double probe electrode is limited to the ion saturation current. For the temperatures and number densities expected $\left(\sim 10-50 \mathrm{eV}, 1-5 \times 10^{18} \mathrm{~m}^{-3}\right)$, this is on the order of several 10-100 microamps, which can be 
quite difficult to measure accurately. This is particularly difficult in chambers as large as the LVTF because the large line length between the probe and external data acquisition system introduces a large amount of capacitance ${ }^{4}$. The current magnitude, of course, depends on the probe size as well. Therefore, the cylindrical probe was chosen to maximize the ion saturation current.

The magnetic field in the thruster influences probe measurements because it constrains the motion of the charged particles. For the conditions in the P5, the ions are essentially unmagnetized since their gyroradii are much larger than the probe dimensions. The electrons, on the other hand, have much smaller gyroradii owing to their much smaller mass. The magnetic field effect manifests itself in two ways; it reduces the electron saturation current $^{5}$ and causes anisotropy of the electron energy distribution function (EEDF) ${ }^{6}$. This anisotropy refers to the fact that, in a sufficiently strong magnetic field, the electrons are observed to have a different measured temperature depending on the probe's orientation relative to the magnetic field. If the probe is oriented such that its collection surface is perpendicular to the magnetic field, it will collect electrons whose motion is chiefly parallel to, and thus not constrained by, the magnetic field. For a probe oriented parallel to the magnetic field, the collected electrons are mainly those whose motion perpendicular to the magnetic field is constrained. Reduction of the electron saturation current causes the electron number density to be substantially underpredicted. This is not an issue with the double probe as number density is inferred from the ion saturation current. Electron temperature can still be determined by standard theory from the slope of the I-V characteristic. However, care must be taken to distinguish between whether this is a parallel, $T e_{\|}$, or perpendicular, $T e_{\perp}$, electron temperature, depending on the orientation of the probe used.

The question of EEDF anisotropy has been investigated by Passoth ${ }^{5}$ and was shown to depend not on the magnetic field, $B$, itself but on the ratio $B / p_{o}$, where $p_{o}$ is the pressure in the containment vessel. Higher pressure effectively increases the number of electron-neutral collisions, randomizing electron motion and reducing any anisotropy induced by the presence of a magnetic field. It has been shown experimentally by Aikawa ${ }^{6}$ that, at a vessel pressure of $1.2 \times 10^{-4}$ Torr, anisotropy of the EEDF was negligible for $B \leq 300 \mathrm{G}$, or $B / p_{o} \leq 2.5 \times 10^{6} \mathrm{G} /$ Torr. In the P5, B has a maximum value of $200 \mathrm{G}$ and the pressure in the discharge chamber is estimated to be approximately $1 \times 10^{-3}$ Torr. This yields a value of $B / p_{o} \leq 2 \times 10^{5} \mathrm{G} / \mathrm{T}$ orr for these experiments, an order of magnitude less than the threshold established by Aikawa. Therefore, there was expected to be no substantial anisotropy in the EEDF in this experiment and thus a single measured electron temperature.
The final consideration in probe design was the orientation of the electrode axis. If the probe axis is aligned with the thruster axis, the electrode could see a potential gradient along its length where the electric field is large. This distorts the probe characteristic, introducing a rounding of the "knee" at the space potential". However, as previously noted, the double probe does not rely on measuring the electron saturation current so this effect is negligible. Alignment with the thruster axis also aligns the axis of the probe with the flowing ions and introduces the problem of "end effects". "End effects" can be minimized by making the probe length to diameter ratio sufficiently large. This alignment has the added benefit of orienting the probe perpendicular to the radial magnetic field, which further minimizes the effect of the magnetic field on electron collection ${ }^{5}$. Conversely, by orienting the probe axis perpendicular to the flow, the high-speed ions distort the electron sheath and the electron temperature can no longer be determined by standard theory?

A final consideration was whether to use a symmetric or asymmetric double probe. The asymmetric probe, with one electrode much larger than the other, would enable data sampling through a larger portion of the EEDF. This could theoretically provide an improved estimate of the electron temperature. However, this would also result in a larger current collection and subsequent perturbation to the plasma. Further, a single equation cannot be fit to the entire current-voltage characteristic for an asymmetric probe, increasing dramatically the difficulty of data reduction. It was decided that the difficulties inherent to the asymmetric probe more than outweighed the potential benefits.

In summary, it was determined that a symmetric, double, cylindrical probe aligned with the axis of the thruster essentially eliminates the problem of the magnetic field effect on electron collection. Several probes were built and tested and the various parameters adjusted as more experience was gained. The final iteration is presented in Figure 4.

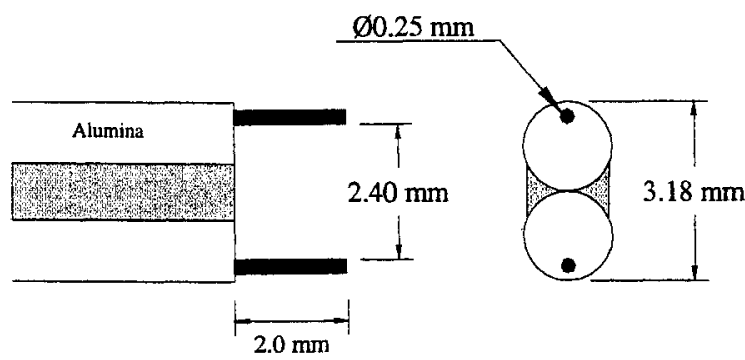

Figure 4. The double cylindrical floating probe consisted of two tungsten electrodes $0.25 \mathrm{~mm}$ in diameter, $2 \mathrm{~mm}$ long, and separated by a distance of $2.4 \mathrm{~mm}$. The alumina insulator had a width of 3.18 mm. 
Each tungsten electrode had a diameter of $0.25 \mathrm{~mm}$ and a length of $2.0 \mathrm{~mm}$. The electrodes were insulated from each other and the plasma by $1.59 \mathrm{~mm}$ diameter alumina tubing and cemented in place with an alumina adhesive. The dimensions were chosen to balance the need for sufficiently large collected currents and length to diameter ratios (larger electrodes), with the need for sufficient resolution and minimal thruster perturbation (smaller electrodes).

As mentioned earlier, one difficulty with the double probe is that it requires a voltage sweep to determine the $\mathrm{I}-\mathrm{V}$ characteristic. Under less demanding circumstances, this can be accomplished with a bi-polar power supply and function generator. The difficulty with this type of setup is that the probe, and hence any required circuitry, is required to float. The floating potential can swing several hundred volts, in a matter of milliseconds, as the probe is moved into the channel. This creates a problem for all of the electronics and can result in significant errors in the measured current if there is any appreciable stray capacitance in the circuit. This problem was minimized by careful attention to the circuit construction to minimize stray capacitance and by using batteries to supply the bias voltage. A potentiometer was attached to the battery output so that the bias could be adjusted. The probe and circuit were isolated from the data collection equipment by two AD210 isolation amplifiers. Figure 5 shows the probe circuit.

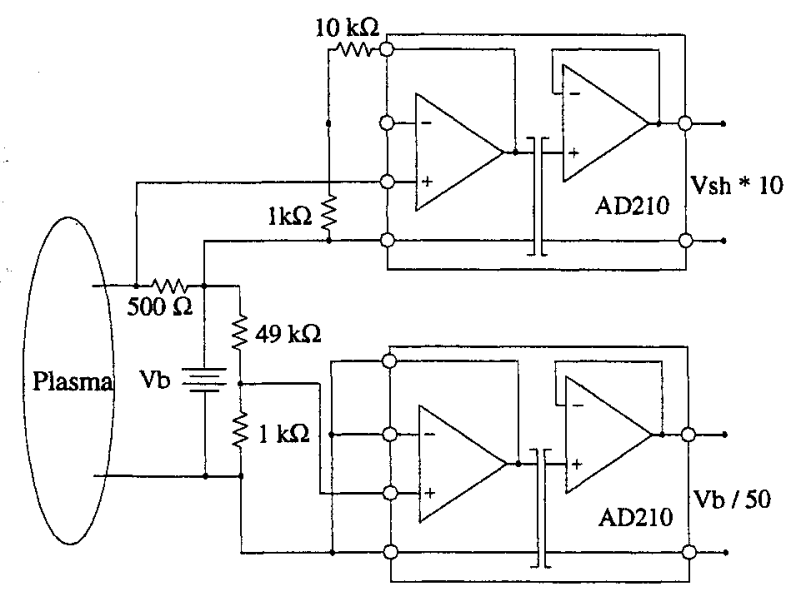

Figure 5. Floating double probe circuit. Batteries supplied the bias between the electrodes and a pair of high common mode voltage isolation amplifiers isolated the circuit from the data acquisition hardware.

For each fixed bias point, the probe current and voltage were measured at each spatial location in the $2 \mathrm{D}$ domain of Figure 3 . The resulting data were then reassembled to get a single current-voltage trace at each point in the domain. Figure 6 shows a representative double probe trace $32 \mathrm{~mm}$ from the anode ( $6 \mathrm{~mm}$ inside the discharge channel) at the center of the channel for a thruster operating condition of $300 \mathrm{~V}$ and $10 \mathrm{~A}$.

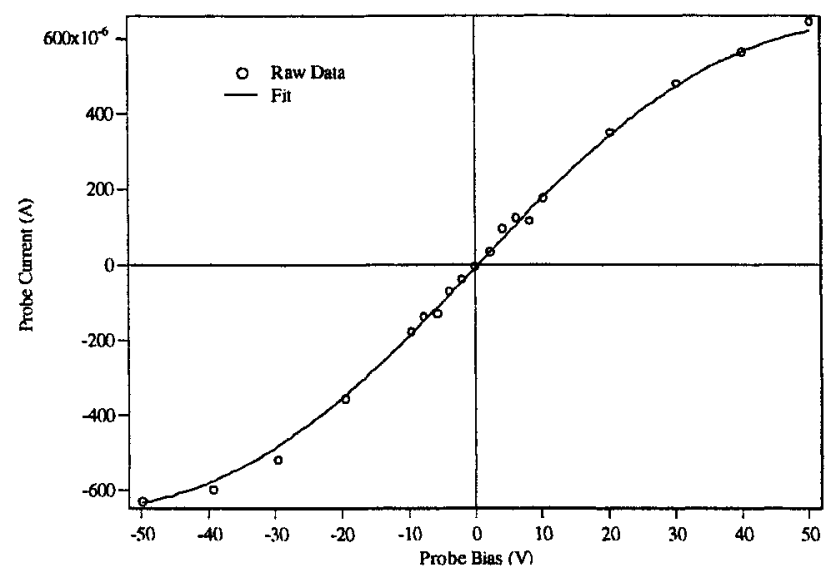

Figure 6. Representative probe characteristic at an axial location of $32 \mathrm{~mm}$ and a radial position of $12.5 \mathrm{~mm}$ (discharge chamber centerline).

In order to obtain the electron temperature and ion number density, the data were fit to the theoretical current-voltage expression for a symmetric double probe ${ }^{4}$ in Equation 5.

$$
I=I_{s a t} \cdot \tanh \left(\frac{\phi}{2 T_{e v}}\right)+A_{1} \cdot \phi+A_{2} \quad \text { Eq. } 5
$$

Here, $T_{e V}$ is the electron temperature in $\mathrm{eV}, I_{\text {sat }}$ is the ion saturation current to either probe, the parameter $A_{1}$ accounts for sheath expansion in the ion saturation region, and the parameter $A_{2}$ reflects any offset current due to stray capacitance ${ }^{4}$, typically on the order of $50 \mu \mathrm{A}$. The scientific graphing package Igor ${ }^{\mathrm{TM}}$ was used to fit this equation to the experimental data using a LevenbergMarquardt method. Ion number density was then calculated from Equation 6, using the $\mathrm{Bohm}^{7}$ approximation for ion velocity.

$$
n_{i}=\frac{I_{s a t}}{A_{s}\left(q Z_{i}\right)^{3 / 2}}\left(\frac{M_{i}}{T_{e v}}\right)^{1 / 2} \quad \text { Eq. } 6
$$

Here, $A_{s}$ is the ion collection area and is initially considered to be equal to the probe surface area. In reality, 
the true collection area depends on the thickness of the sheath surrounding the probe, which is determined through an iterative process. Having calculated the temperature and initial number density, the sheath thickness is calculated according to Equation $7^{8}$ :

$$
\begin{gathered}
\delta=1.02 \lambda_{D}\left[\left(\frac{1}{2} \ln \left(\frac{m}{M}\right)\right)^{1 / 2}-\frac{1}{\sqrt{2}}\right]^{1 / 2} . \\
{\left[\left(\frac{1}{2} \ln \left(\frac{m}{M}\right)\right)^{1 / 2}+\sqrt{2}\right]}
\end{gathered}
$$

Eq. 7

where $\lambda_{D}$ is the Debye shielding length for electrons. It follows that the sheath area is then

$$
A_{s}=A_{p}\left(1+\frac{\delta}{r}\right)
$$

Eq. 8

where $A_{s}$ is the area of the sheath, $A_{p}$ is the area of the probe, and $r$ is the radius of the probe. With the corrected area for the sheath, the number density is re-calculated. This process is repeated until the number density converges to a final solution, typically $10-15$ iterations.

The above analysis assumes that the "end effects" are negligible based on the dimensions and orientation of the probe electrodes. Using the plasma potential measurements from the emissive probe, the axial ion velocity can be estimated and the effect of the directed ion flux to the tip of the probe included. This is accomplished by assuming quasi-neutral $\left(n_{i}=n_{e}\right)$, steady, onedimensional flow in the discharge channel. Thus the continuity equations for ions and neutrals can be written as:

$$
\begin{array}{ll}
\nabla \cdot\left(n_{i} \vec{V}_{i}\right)=\kappa\left(T_{e v}\right) n_{i} n_{n} & \text { Eq. } 9 \\
\nabla \cdot\left(n_{n} \vec{V}_{n}\right)=-\kappa\left(T_{e v}\right) n_{i} n_{n} & \text { Eq. } 10
\end{array}
$$

and the momentum equations as:

$$
\nabla \cdot\left(n_{i} \vec{V}_{i} \vec{V}_{i}\right)=\frac{q E_{z} n_{i}}{M}+\kappa\left(T_{e v}\right) n_{i} n_{n} \vec{V}_{i} \quad \text { Eq. } 11
$$

$$
\nabla \cdot\left(n_{n} \vec{V}_{n} \vec{V}_{n}\right)=-\kappa\left(T_{e v}\right) n_{i} n_{n} \vec{V}_{n} \quad \text { Eq. } 12
$$

Combining the above equations, and using a forward differencing discretization, the set is reduced to two equations:

$$
\left.n_{i} V_{i}\right|_{k}+\left.n_{n} V_{n}\right|_{k}=\left.n_{i} V_{i}\right|_{k+1}+\left.n_{n} V_{n}\right|_{k+1}
$$

$$
\frac{\left.n_{i} V_{i}^{2}\right|_{k+1}-\left.n_{i} V_{i}^{2}\right|_{k}}{\Delta z}+\frac{\left.n_{n} V_{n}^{2}\right|_{k+1}+\left.n_{n} V_{n}^{2}\right|_{k}}{\Delta z}
$$

Eq. 14

$$
=\frac{q E_{z}}{M} \frac{1}{2}\left(\left.n_{i}\right|_{k+1}+\left.n_{i}\right|_{k}\right)
$$

In Equations 13 and $14, n_{i}$ and $E_{z}$ are known quantities from probe measurements, as are the initial conditions at the rear of the channel. $V_{i}$ and $n_{n}$ are calculated by stepping sequentially through each axial position.

Once the ion velocity has been estimated, the ion number density can be re-calculated taking into account the flux of ions to the probe tip. The area used for this portion of the collected ion current, $A_{d}$, includes the probe tip area as well as the portion of the sheath in which entering ions are collected before they bypass the electrode ${ }^{7}$ :

$$
A_{d}=\pi\left(r+\frac{Z^{1 / 2} l}{V_{i}}\left(\frac{T_{e v}}{M}\right)^{1 / 2}\right)^{2}
$$

$$
n_{i}=\frac{I_{s a t}}{A_{s}(q Z)^{3 / 2}\left(\frac{T_{e v}}{M_{i}}\right)^{1 / 2}+q Z V_{i} A_{d}}
$$

Eq. 16 
Combining this with the sheath calculation, the ion number density calculation can be iterated until it converges to a final solution.

\section{RESULTS AND DISCUSSION}

\section{Emissive and Langmuir Probe Results}

Plasma potential, ion number density, and electron temperature were measured in the discharge channel of the P5 and have been discussed in detail in previous works ${ }^{9,10}$; the contour plots are presented below in Figures $17-22$ for reference during discussion of the Hall current.

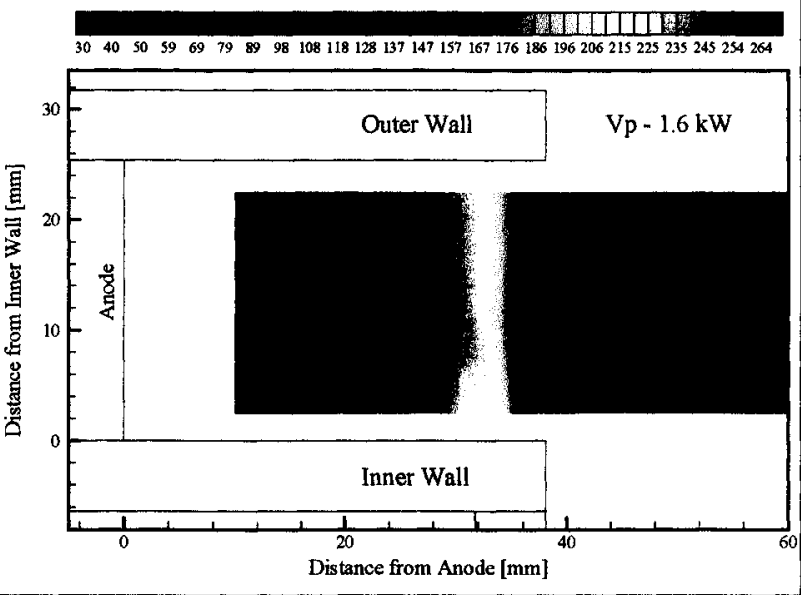

Figure 17. Plasma potential contours at $1.6 \mathrm{~kW}$. Units are in volts.

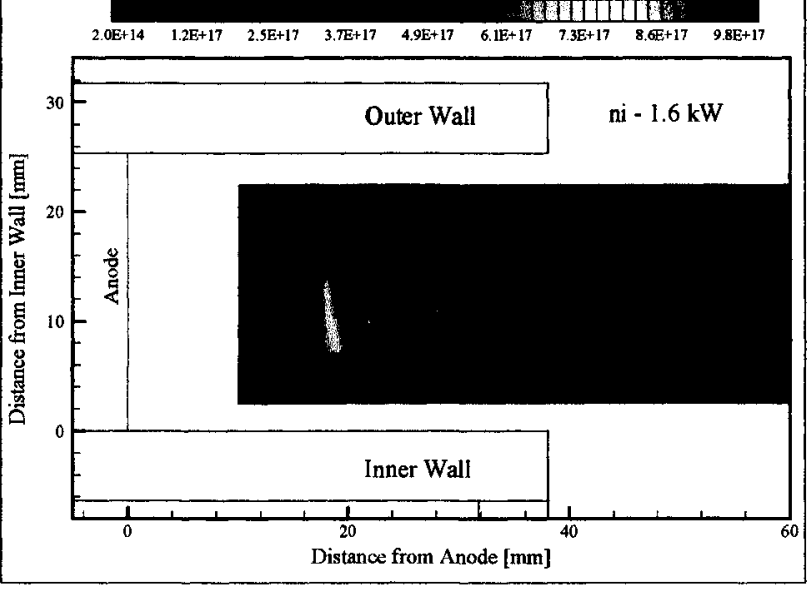

Figure 18. Ion number density contours, $1.6 \mathrm{~kW}$. Units are in $\mathbf{m}^{-3}$.

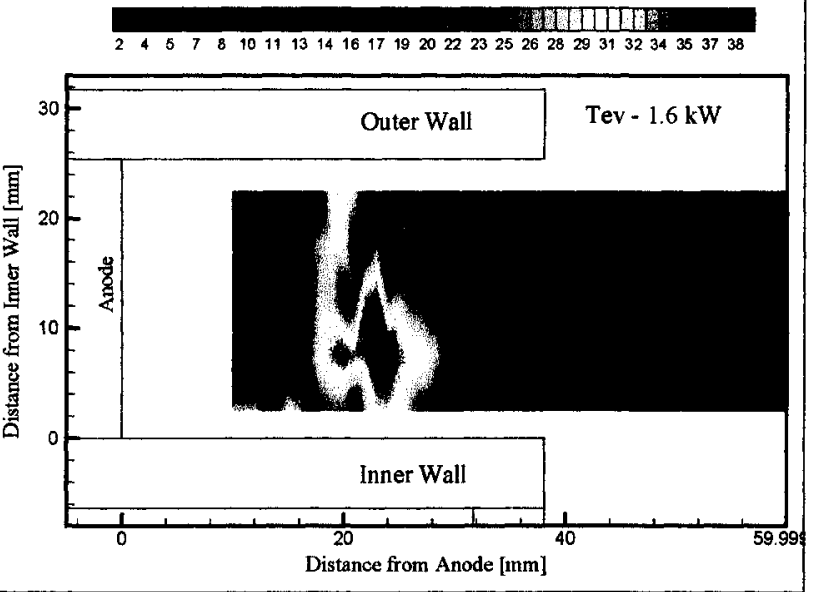

Figure 19. Electron temperature contours, $1.6 \mathrm{~kW}$. Units are in $\mathrm{eV}$.

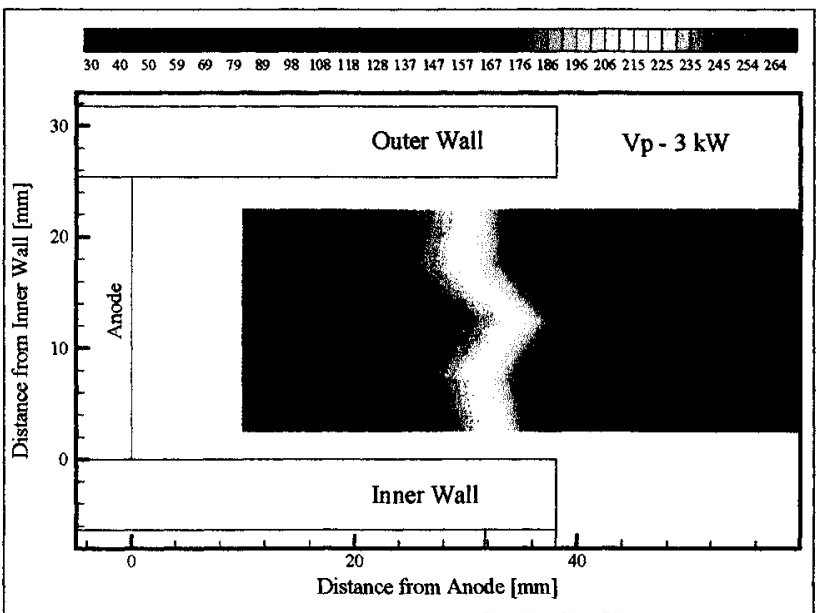

Figure 20. Plasma potential contours at $3 \mathrm{~kW}$. Units are in volts.

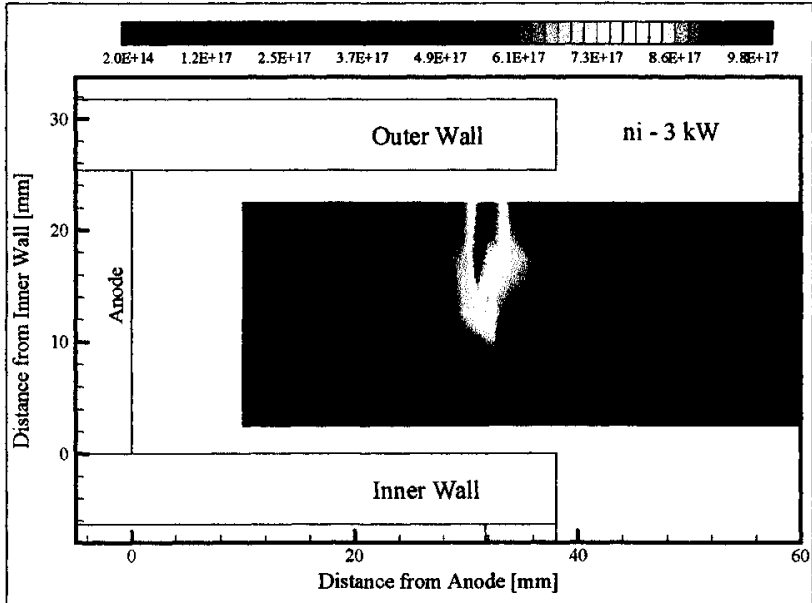

Figure 21. Ion number density contours, $3 \mathrm{~kW}$. Units are in $\mathbf{m}^{-3}$. 


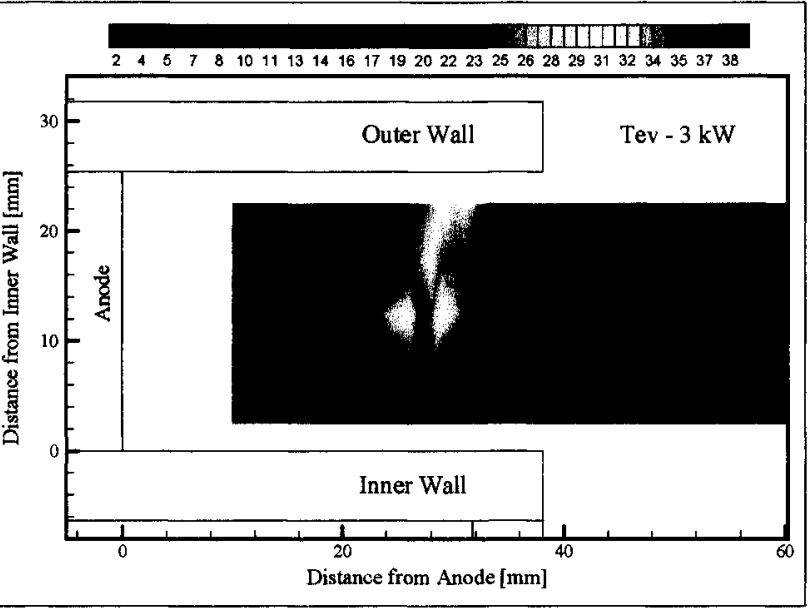

Figure 22. Electron temperature contours, 3 kW. Units are in $\mathrm{eV}$.

The ion number density contour plot of Figure 18 illustrates the most striking feature of the $1.6 \mathrm{~kW}$ operating condition. The data exhibit, with the exception of the inner wall region, a double-axial-peak structure indicating two regions of ionization. The primary zone occurs quite far upstream of the exit plane $(\sim 20 \mathrm{~mm})$ while the second occurs much closer to the exit plane $(-32 \mathrm{~mm})$. Referring to Figure 19, it can be seen that this primary ionization zone closely corresponds spatially with the peak electron temperature. The electron temperature begins to decrease considerably in the vicinity of the secondary peak. Further, Figure 17 shows that the potential is monotonically decreasing in the axial direction, thus there is no deceleration of ions that might explain the local increase in number density.

In contrast to the $1.6 \mathrm{~kW}$ case, data at $3 \mathrm{~kW}$ show a single ionization zone near the exit plane, at the beginning of the acceleration region. The axial number density profiles closely follow the electron temperature, which is expected, as the high-temperature electrons are the primary ionizing mechanism.

\section{Hall Current Density}

Using data obtained from the emissive and double Langmuir probes, the Hall current density is computed according to Equations 2 and 3 . The results for the $1.6 \mathrm{~kW}$ and $3 \mathrm{~kW}$ cases are presented below in Figures 23 and 24, respectively.

In general, the two cases display very similar results. The bulk of the Hall current is confined to a region centered several millimeters upstream of the exit plane, is of the same order of magnitude, and is asymmetric about the centerline of the discharge channel. The asymmetry is a consequence of the magnetic field topology, shown in Figure 25 for the $3 \mathrm{~kW}$ case.

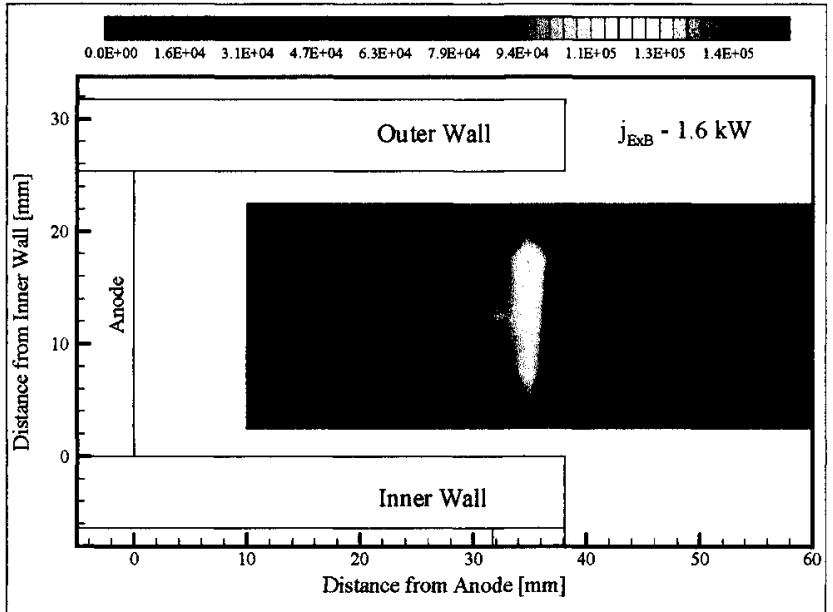

Figure 23. Hall current density calculated from measured electric and magnetic fields, $1.6 \mathrm{~kW}$. Units are in $\mathrm{A} / \mathrm{m}^{2}$.

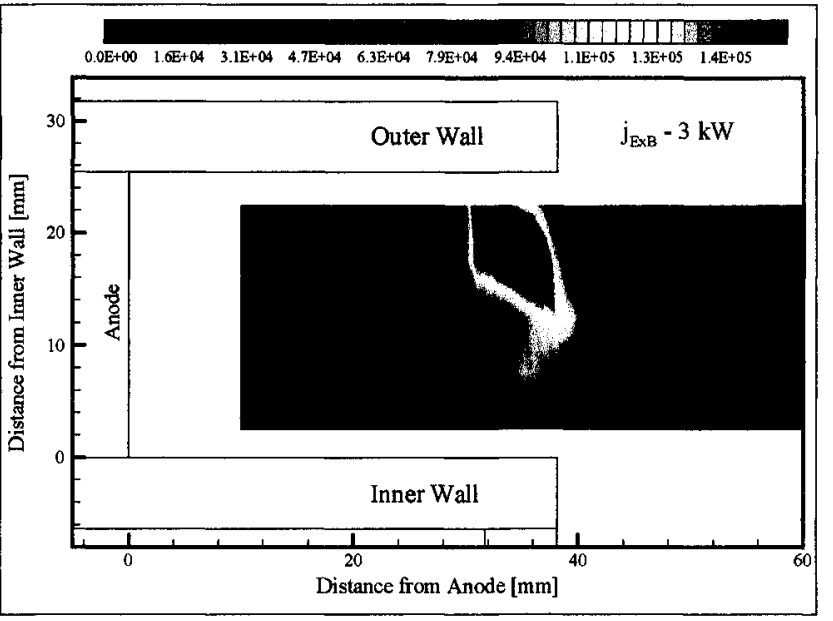

Figure 24. Hall current density calculated from measured electric and magnetic fields, $3 \mathrm{~kW}$. Units are in $A / \mathbf{m}^{2}$.

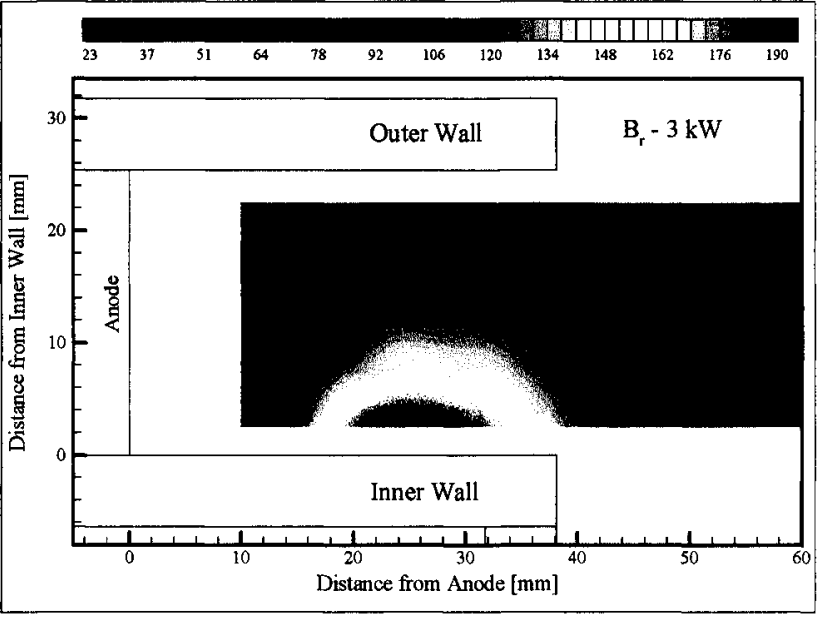

Figure 25. Radial magnetic field contours, $3 \mathrm{~kW}$. Units are in Gauss. 
The magnetic field contours are essentially the same for both operating conditions, differing only in that the magnitude at $1.6 \mathrm{~kW}$ is approximately $10 \%$ lower.

The main difference between the two operating conditions lies in the axial extent of the Hall current; At $1.6 \mathrm{~kW}$, the axial plasma potential drop occurs over a much shorter distance, resulting in a more sharply peaked Hall current zone, as compared to the $3 \mathrm{~kW}$ case.

One of the key questions arising from the electron temperature and number density data is the cause of the double peaked structure in the axial number density profile at $1.6 \mathrm{~kW}$. As discussed earlier, the first peak at 20 $\mathrm{mm}$ corresponds to a region of high-temperature electrons.

Looking at Figure 23, it can be seen that the secondary ionization zone corresponds closely to the location of the Hall current zone. Having determined the electron drift velocity in the channel, an equivalent electron kinetic energy can be computed, the axial profiles of which are shown in Figure 26, for each radial location.

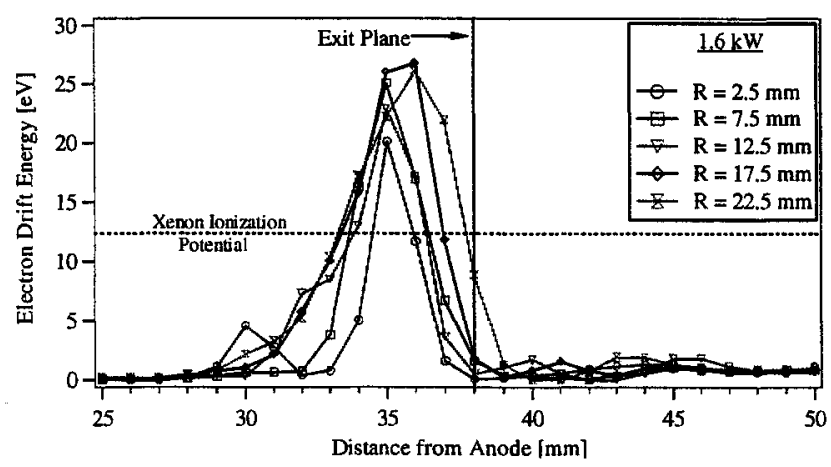

Figure 26. Hall current density calculated from measured electric and magnetic fields, $1.6 \mathrm{~kW}$. Units are in $\mathrm{A} / \mathrm{m}^{2}$.

From Figure 26, it is clear that the electron drift energy is well above the ionization potential for Xenon. This strongly suggests the secondary ionization zone is due to a population of electrons with a large drift velocity, i.e. the Hall current.

The contribution of the Hall current to ionization is not as obvious in the $3 \mathrm{~kW}$ case. The electron temperature, ion number density and Hall current each exhibit a single axial peak across the width of the channel, making it difficult to distinguish between the high-temperature and high-drift velocity electrons. The exception is the centerline data. Figure 27 shows axial profiles of the electron temperature and ion number density for the $3 \mathrm{~kW}$ case.

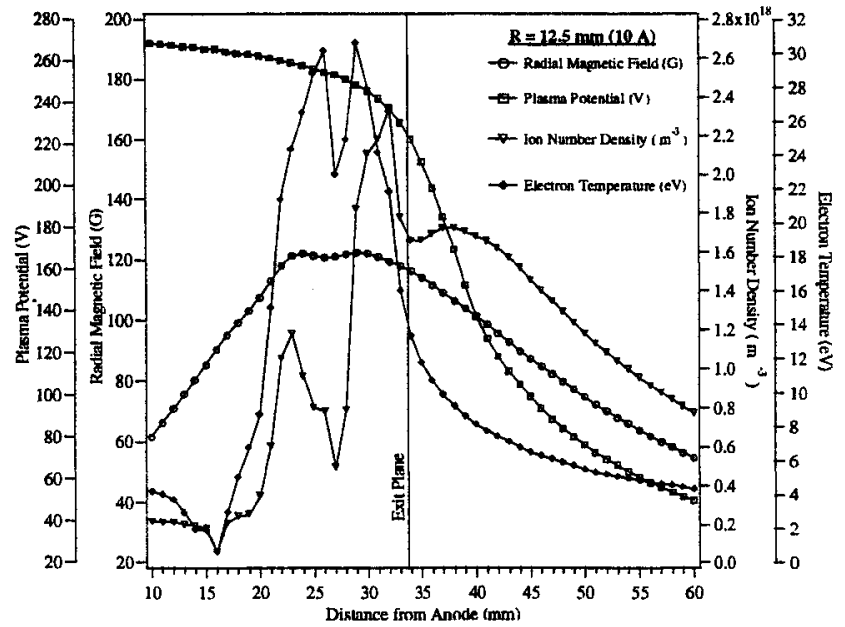

Figure 27. Plasma potential, radial magnetic field, electron temperature, and ion number density, $3 \mathrm{~kW}, \mathrm{R}=12.5 \mathrm{~mm}$.

Here, the ion number density has a small secondary peak at the exit plane. Looking at the electron drift energy, Figure 28 , there is a corresponding peak in the drift energy at the exit plane on centerline of the discharge channel, again suggesting ionization from the azimuthally drifting electrons.

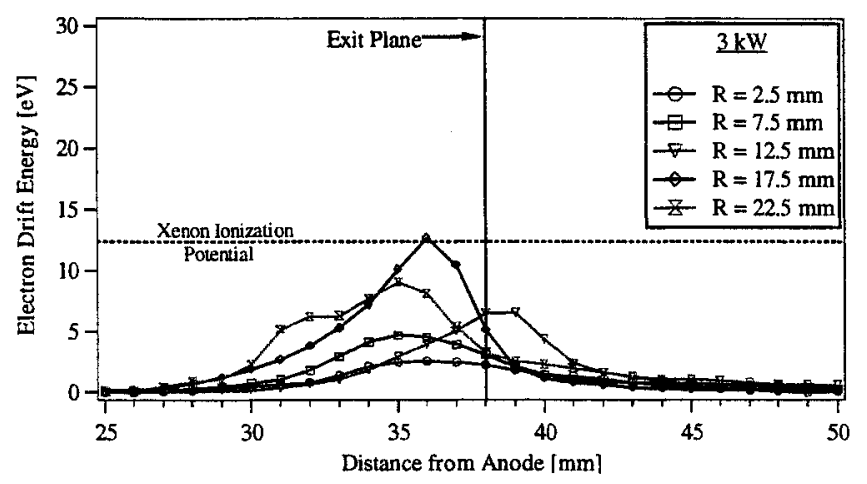

Figure 28. Hall current density calculated from measured electric and magnetic fields, $1.6 \mathrm{~kW}$. Units are in $\mathrm{A} / \mathrm{m}^{2}$.

However, this raises an additional issue; namely, the drift energy of the electrons at $3 \mathrm{~kW}$ is well below the ionization potential for Xenon and would not ionize the neutral particles. The key to addressing this apparent inconsistency is to consider the relative velocities of the electrons and neutrals. The azimuthal electron drift velocity is an order of magnitude greater than the axial velocity of the relatively slow moving ions. Thus, the electrons will effect many rotations about the circumference of the discharge channel in the time it takes the ions to transit the Hall current region. This greatly increases the probability that the ions will undergo multiple collisions with the drifting electrons and be 
ionized. Therefore, at both operating conditions the Hall current acts like an electron "buzz saw" near the exit plane, ionizing Xenon neutrals.

\section{Thrust}

Given the presence of both electric and magnetic fields in the Hall thruster, the question of whether plasma acceleration is electrostatic (i.e., electric body forces) or electromagnetic (i.e., interaction of magnetic fields and plasma currents) is often raised. Using the Hall current density, the electromagnetic body force on the ions can be calculated and compared to the total thrust from a thrust stand.

The MHD equations are an ideal starting point for the electromagnetic analysis of the Hall thruster as they describe the interaction of the currents driven in the plasma with the applied magnetic field. The MHD momentum equation, also called the force equation, expresses the volumetric body force $(F / \forall)$ on the plasma and is given by Equation 17 .

$$
\mathbf{F}=-\nabla P+\rho_{c} \mathbf{E}+\mathbf{j} x \mathbf{B} \quad \text { Eq. } 17
$$

The electron pressure term is small compared to the $\mathbf{j} x \mathbf{B}$ term over most of the discharge channel. Additionally, the quasineutrality assumption means there is no net charge imbalance $\left(\rho_{c}=0\right)$. Therefore, the axial body force density can be written

$$
\frac{F_{z}}{\forall}=-j_{\theta} B_{r}
$$

As in the previous section, note that the ions are unmagnetized; thus the azimuthal current density in Equation 18 is composed entirely of the drifting electrons. Rewriting the current density according to Equation 3 and recalling that the electron drift velocity is simply $V_{E x B}=E_{z}$ $/ B_{r}$, the body force density is

$$
\frac{F_{z}}{\forall}=-q n_{i} E_{z}
$$

Summing over each volumetric element in the thruster discharge and noting that, for electrons, $-q=+|q|$, the total thrust can be written

$$
T=\sum_{k}|q| n_{i}^{k} E_{z}^{k} \forall^{k}
$$

Conversely, one can consider the electrostatic acceleration of the plasma. This motion is described by the Lorentz force equation, Equation 6-30.

$$
\mathbf{F}=q(\mathbf{E}+\mathbf{V} x \mathbf{B}) \quad \text { Eq. } 21
$$

Making the simplifying assumption that the magnetic field is radial and the electric field is axial ${ }^{\S}$, the component of force directed along the thruster axis can be written

$$
F_{z}=q\left(E_{z}-V_{\theta} B_{r}\right) \quad \text { Eq. } 22
$$

The assumption that the net force on the electrons is zero, from which the $\operatorname{ExB}$ drift velocity, $V_{E x B}=E_{z} / B_{r}$, is derived, can be justified as follows. The electron drift is a cumulative effect over many gyroradii. During each gyroperiod, the electron experiences acceleration in a given direction over part of its orbit, which reverses as it gyrates about the magnetic field. As such, over many gyroperiods, the averaged acceleration, and hence the net force, is zero. The ions, on the other hand, are essentially unmagnetized, having gyroradii larger than the discharge channel dimensions. As a result, they develop no azimuthal motion. Thus the force on a single ion is simply

$$
F_{z}=|q| E_{z} \quad \text { Eq. } 23
$$

The thrust is then the sum of the force on all the ions in each volumetric element, $k$, throughout the thruster discharge.

$$
T=\sum_{k}|q| n_{i}^{k} E_{z}^{k} \forall^{k}
$$

Eq. 24

This is precisely the same as the result of the electromagnetic analysis in Equation 20. Table 1 compares thrust values obtained with a NASA-style, inverted-pendulum thrust stand and those calculated using the Hall current density integrated over the plasma volume. Two numbers are listed for the calculated thrust values:

\footnotetext{
$\S$ Strictly speaking, this is not true throughout the entire discharge channel and near-field region of the thruster. However, the majority of the acceleration is confined to a region centered several millimeters upstream of the exit plane and in this region the magnetic and electric fields are essentially radial and axial, respectively.
} 
thrust generated inside the discharge channel and the total thrust.

Table 1. Comparison of measured and calculated thrust.

\begin{tabular}{|c|c|c|c|c|}
\hline \multirow{2}{*}{\multicolumn{2}{|c|}{ 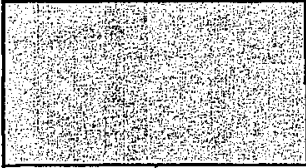 }} & \multicolumn{2}{|c|}{$T=j x B=n_{i} q E \psi$} & \multirow{2}{*}{ 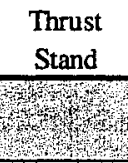 } \\
\hline & & $\begin{array}{c}38 \mathrm{~mm} \\
\text { (Exit Plane) }\end{array}$ & $60 \mathrm{~mm}$ & \\
\hline \multirow[t]{2}{*}{$1.6 \mathrm{~kW}$} & Thrust $[\mathrm{mN}]$ & 68 & 93 & $\begin{array}{c}95 \\
\pm 3 \mathrm{mN}\end{array}$ \\
\hline & $\begin{array}{c}\text { Total Thrust } \\
\text { Fraction }\end{array}$ & 0.72 & 0.98 & - \\
\hline \multirow[t]{2}{*}{$3 \mathrm{~kW}$} & Thrust [mN] & 125 & 168 & $\begin{aligned} & 175 \\
\pm & 3 \mathrm{mN}\end{aligned}$ \\
\hline & $\begin{array}{c}\text { Total Thrust } \\
\text { Fraction }\end{array}$ & 0.71 & 0.96 & - \\
\hline
\end{tabular}

The results show excellent agreement between the calculated and measured total thrust. At both power levels, the results agree to within 5\%. Further, the Hall current calculation affords the ability to examine where thrust is generated in the plasma discharge; comparing the values at $38 \mathrm{~mm}$ and $60 \mathrm{~mm}$ (where the Hall current has dropped to negligible levels), it is shown that nearly $30 \%$ of the thrust is generated outside the discharge channel.

\section{Self-Magnetic Field Calculations}

The self-field generated by the Hall current is of particular interest as it may significantly alter the magnetic field structure and hence the operation of the thruster ${ }^{11}$. Thus, Hall current density distributions derived from probe measurements were used to estimate the influence of the self-field to the applied field. The discharge channel was divided into a rectangular mesh with a cell size of $1 \mathrm{~mm}$ by $5 \mathrm{~mm}$. The Hall current in a given cell was computed using the current density from Figures 23 and 24 and the cell area. The Hall current in a given cell was approximated as current flowing in a long straight wire allowing the self-field to be computed using the BiotSavart law shown in Equation 17

$$
\mathbf{B}=\frac{\mu_{0} \mathbf{I}}{2 \pi r_{I}}
$$

where $\mathbf{B}$ is the resulting self-field, I is the Hall current, and $r_{I}$ is the distance between the approximated wire and another point in the computational domain. The resulting self-field was decomposed into its axial and radial components. Figure 29 illustrates the domain.

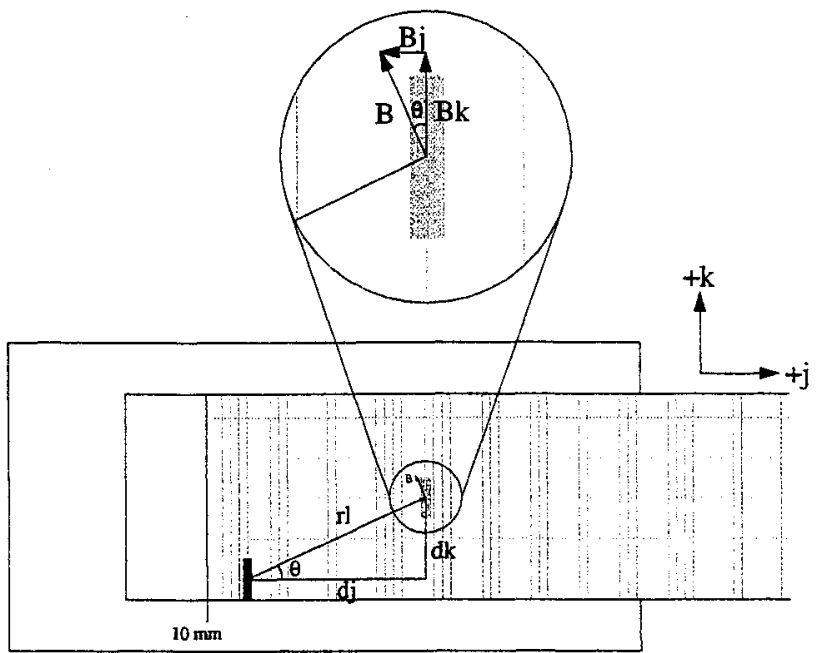

Figure 29. Calculation of self magnetic fields in the P5 discharge channel.

An iterative approach was used to obtain the self-field throughout the domain. The static magnetic field and electric field were used to compute an initial Hall current. This current was used to compute the resulting self-field in the plasma. The self-field was then added to the static magnetic field and the Hall current recalculated. This process was repeated until the calculated self-field converged. Figures $30-33$ show the computed axial and radial magnetic self-fields for the 1.6 and $3 \mathrm{~kW}$ cases.

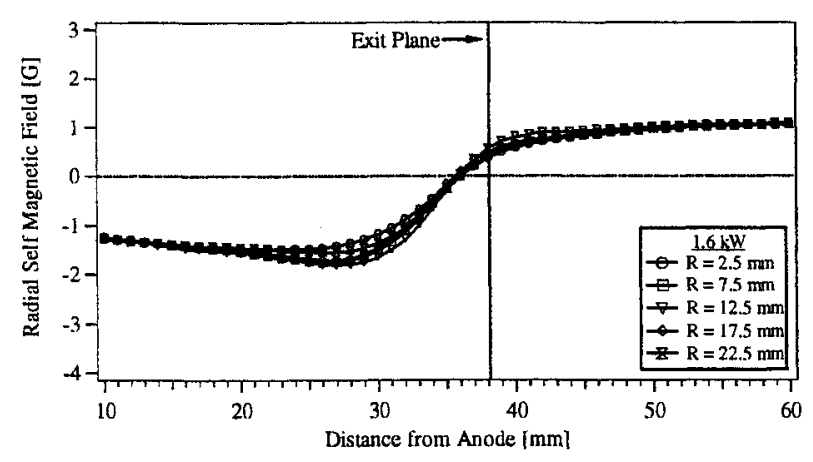

Figure 30. Radial self magnetic field from computed Hall current, $1.6 \mathrm{~kW}$. 


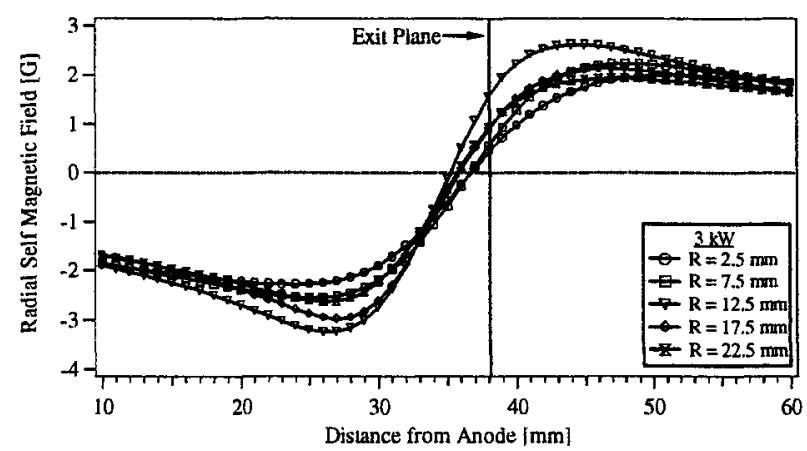

Figure 31. Radial self magnetic field from computed Hall current, 3 kW.

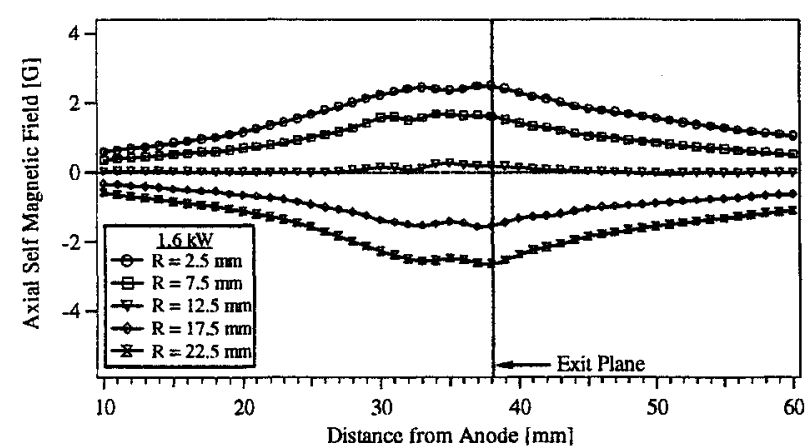

Figure 32. Axial self magnetic field from computed Hall current, 1.6 kW.

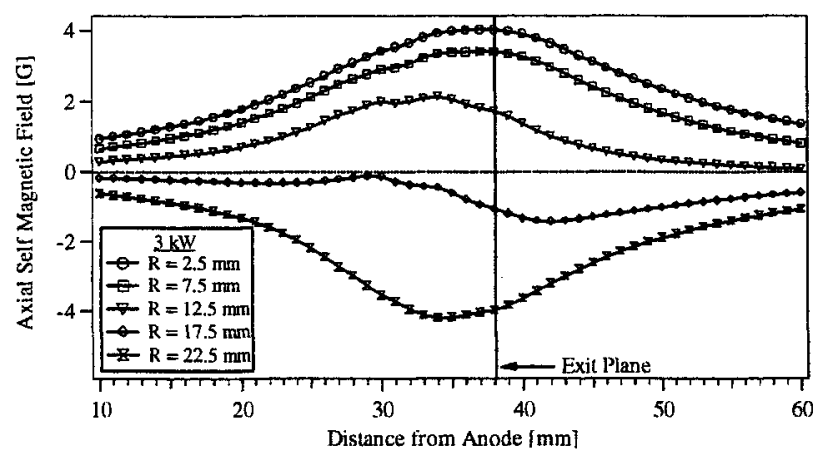

Figure 31. Axial self magnetic field from computed Hall current, 3 kW.

The results of the self-field calculations show that the Hall current does not contribute any substantial change to the static field; the axial and radial self-field magnitudes did not exceed several Gauss. This was negligible compared to the applied fields, which were several hundred Gauss. However, the contribution of the self-field may change considerably at other power levels. Table 2 shows the total integrated Hall current at the two power levels under consideration.
Tahle 2. Total Hall current and its comparison to thruster discharge current.

\begin{tabular}{|c|c|c|c|}
\hline \multicolumn{2}{|c|}{} & $\begin{array}{c}38 \mathrm{~mm} \\
\text { (Exit Plane) }\end{array}$ & $60 \mathrm{~mm}$ \\
\hline $1.6 \mathrm{~kW}$ & Hall Current [A] & 14.6 & 25.1 \\
\hline & Current Ratio $\left(\mathrm{I}_{\mathrm{Hal}} / \mathrm{I}_{\mathrm{D}}\right)$ & 2.7 & 4.6 \\
\hline $3 \mathrm{~kW}$ & Hall Current $[\mathrm{A}]$ & 23.3 & 34.6 \\
\hline & Current Ratio $\left(\mathrm{I}_{\mathrm{Hal}} / \mathrm{I}_{\mathrm{D}}\right)$ & 2.3 & 3.5 \\
\hline
\end{tabular}

The key value in Table 2 is the current ratio; this shows that the Hall current is $3.5-4.6$ times larger than the thruster discharge current. As Hall thrusters are scaled up in power, more specifically to higher current, the Hall current is expected to increase to hundreds of amps. At these levels, the Hall current may significantly alter the configuration of the magnetic field during thruster operation; particularly since scaling laws suggest that the applied magnetic field will drop with increasing thruster size $e^{12}$.

\section{Acknowledgement}

The authors wish to thank Rafael Martinez for making the magnetic field maps of the P5 and the University of Michigan Undergraduate Research Opportunity Program (UROP) for his support. The authors would also like to acknowledge the fellow researchers and technicians at PEPL and of the Department of Aerospace Engineering, respectively. This research was supported through a grant from the Air Force Office of Scientific Research (F4962098-1-0033-Dr. M. A. Birkan, program manager) and by the Palace Knight program.

\section{REFERENCES}

1 Hershkowitz, N., Cho, M., "Measurement of Plasma Potential Using Collecting and Emitting Probes," Journal of Vacuum Science \& Technology A, Vol. 6, No. 3, May 1988, p. 2054.

${ }^{2}$ Tilley, D.L., et. al., "The Application of the Triple Probe Method to MPD Thruster Plumes," AIAA 90-2667, 26 ${ }^{\text {th }}$ Joint Propulsion Conference, July 1990. 
${ }^{3}$ Haas, J.M., Gallimore, A.D., "Characterization of the Internal Plasma Structure of a $5 \mathrm{~kW}$ Hall Thruster," IEPC99-078, 26th International Electric Propulsion Conference, Kitakyushu, Japan, October 1999.

${ }^{4}$ Smith, B.A., Overzet, L.J., "Improvements to the floating double probe for time-resolved measurements in pulsed if plasmas," Review of Scientific Instruments., Vol. 69, No. 3, p. 1372, March 1998.

${ }^{5}$ Passoth, E., et. al., "An experimental study of plasma density determination by a cylindrical Langmuir probe at different pressures and magnetic fields in a cylindrical magnetron discharge in heavy rare gases," Journal of Physics D., Vol.30, No.12, June 1997, p.1763-77.

${ }^{6}$ Aikawa, H., "The Measurement of the Anisotropy of Electron Distribution Function of a Magnetized Plasma," Journal of the Physical Society of Japan, Vol. 40, No. 6, June 1976.

${ }^{7}$ Chung, P.M., et. al., "Electrostatic Probes in Stationary and Flowing Plasmas: Part 1. Collisionless and Transitional Probes," AIAA Journal, Vol. 12, No. 2, February 1974.

8 Peterson, P.Y., Initial Scoping Studies on Applied Magnetically Distributed Arcs for Plasma Torches, M.S. Thesis, Raleigh, North Carolina: Department of Nuclear Engineering, North Carolina State university, 1996.

9 Haas, J.M., and Gallimore, A.D., "An Investigation of Internal Ion Number Density and Electron Temperature Profiles in a Laboratory-Model Hall Thruster," AIAA 2000-3422, 36 ${ }^{\text {th }}$ Joint Propulsion Conference, July 2000.

${ }^{10}$ Haas, J.M., and Gallimore, A.D., "Characterization of the Internal Plasma Structure of a $5 \mathrm{~kW}$ Hall Thruster," IEPC-99-078, 26 ${ }^{\text {th }}$ International Electric Propulsion Conference, Kitakyushu, Japan, October 1999.

1 Peterson, P., Haas, J., Hofer, R., Gallimore, A., "Experimental Investigation of Hall Thruster Magnetic Field Topography," ICOPS 2000-4A07, 27th IEEE International Conference on Plasma Science, New Orleans, LA, June 2000.

${ }^{12}$ Maslenikov, N.A., Russian Electric Propulsion Seminar, Massachusetts Institute of Technology, 1991. 